\title{
EFEITOS DA ORIENTAÇÃO DA ATIVIDADE FÍSICA EM PACIENTES COM OSTEOARTRITE AVANÇADA DO JOELHO
}

\author{
EFFECTS OF PRESCRIBED PHYSICAL ACTIVITY IN PATIENTS WITH ADVANCED \\ OSTEOARTHRITIS OF THE KNEE
}

Artigo Original

Original ARTICLE

Artículo Original

\author{
EFECTOS DE LA ORIENTACIÓN DE LA ACTIVIDAD FÍSICA EN PACIENTES CON \\ OSTEOARTRITIS DE RODILLA AVANZADA
}

\section{Ricardo de Souza Campos Fernandes ${ }^{1}$ (Médico Ortopedista) Monica Paschoal Nogueira' (Médica Ortopedista)}

1. Hospital do Servidor Público do Estado de São Paulo, Departamento de Ortopedia e Traumatologia, São Paulo, SP, Brasil.

\section{Correspondência:}

Av. Ibirapuera, $981,2^{\circ}$ andar, Vila Clementino, São Paulo, SP, Brasil. 04029-000.

ricfernandes7@hotmail.com

\section{RESUMO}

Introdução: A osteoartrite (OA) do joelho atinge $20 \%$ da população mundial e $70 \%$ a $80 \%$ dos indivíduos com mais de 65 anos. Caracteriza-se por dor e incapacidade funcional determinada pela degeneração da cartilagem articular e do osso subcondral. Seu tratamento conservador inclui a prática de exercícios físicos. Objetivo: Determinar os efeitos da prescrição e orientação da atividade física em pacientes com OA avançada do joelho. Métodos: Foram incluídos 23 pacientes submetidos a atividade física com orientação e avaliados no momento inicial, aos três e aos seis meses, pelo sistema de pontuação EVA e WOMAC (3 domínios). Foi utilizado o modelo linear generalizado e nas situações com diferença significante entre os tempos de avaliação, foram feitas comparações múltiplas com o teste de Bonferroni, com nível de significância de cinco por cento. Resultados: Houve diferença estatisticamente significante entre as pontuações EVA e WOMAC no início do estudo e aos três e seis meses. Esses resultados foram independentes do grau de OA e de doença uni ou bilateral. Conclusão: A orientação da atividade física no tratamento de pacientes com OA avançada de joelho, melhora a função articular e deve ser considerada inclusive nos casos com indicação de artroplastia.

Descritores: osteoartrite; atividade física; joelho; idoso.

\section{ABSTRACT}

Introduction: Osteoarthritis (OA) of the knee affects $20 \%$ of the world's population and $70 \%$ to $80 \%$ of individuals aged over 65 years. It is characterized by pain and functional disability caused by the degeneration of joint cartilage and subchondral bone. Its conservative treatment includes physical exercises. Objective: To determine the effects of the prescription of a specific protocol of physical activities to treat OA in patients with severe OA of the knee. Methods: Twenty-three patients were selected, and physical activities were prescribed. The patients were evaluated at baseline, three and six months with the Visual Analogue Scale (VAS) and Western Ontario and McMaster Universities Osteoarthritis (WOMAC) index (3 domains). The generalized linear model was used, and in situations with significant difference between the evaluation times, multiple comparisons were conducted using the Bonferroni test, with a level of significance of five percent. Results: There were statistically significant differences between the VAS and WOMAC scores at baseline and three and six months. These results were independent of the degree of OA and whether the disease was unilateral or bilateral. Conclusion: Prescription of physical activities in patients with severe OA of the knee improves articular function, and should therefore be considered in cases with indication for arthroplasty.

Keywords: osteoarthritis; motor activity; knee; aged.

\section{RESUMEN}

Introducción: La osteoartritis (OA) de la rodilla alcanza 20\% de la población mundial y 70\% a 80\% de los individuos de más de 65 años. Se caracteriza por dolor y la discapacidad funcional determinada por la degeneración del cartílago articulary del hueso subcondral. Su tratamiento conservador incluye el ejercicio físico. Objetivo: Determinar los efectos de la prescripción y la orientación de la actividad física en pacientes con osteoartritis de rodilla avanzada. Métodos: Se incluyeron 23 pacientes sometidos a la actividad física con orientación y evaluados al inicio del estudio, a los tres y seis meses a través del sistema de puntuación EVA y WOMAC (3 dominios). Se utilizó el modelo lineal generalizado y en situaciones con diferencias significativas entre los tiempos de evaluación, se realizaron múltiples comparaciones con la prueba de Bonferroni, con un nivel de significación del 5\%. Resultados: Hubo diferencia estadísticamente significativa entre las puntuaciones EVA y WOMAC en el inicio del estudio y a los tres y seis meses. Estos resultados fueron independientes del grado de OA y de la enfermedad unilateral o bilateral. Conclusión: La orientación de la actividad física en el tratamiento de pacientes con osteoartritis de rodilla avanzada, mejora la función articular y debe considerarse incluso en casos con indicación de artroplastia.

Descriptores: osteoartritis; actividad motora; rodilla; anciano. 


\section{INTRODUÇÃO}

A osteoartrite (OA) é uma condição clínica comum entre os idosos, como consequência da degeneração da cartilagem articular e do osso subcondral, sendo o joelho, a articulação de suporte de carga mais acometida. Manifesta-se por dor, rigidez articular, incapacidade funcional e fraqueza da musculatura flexora e extensora do joelho, além de distúrbios do alinhamento derivados da perda de cartilagem articular e alterações ligamentares. Frequentemente, determina um impacto negativo na qualidade de vida, perdas econômicas e gastos com saúde..$^{1-3}$

O diagnóstico da OA é realizado a partir dos sintomas clínicos e dos exames radiográficos que mostram alterações ósseas, como osteófitos, esclerose subcondral e diminuição do espaço articular. ${ }^{4}$ A classificação de Ahlbäck modificada por Keyes et al. ${ }^{6}$, divide a OA em cinco graus, de acordo com o comprometimento articular radiográfico.

O tratamento conservador da OA é baseado no controle dos sintomas (dor, redução da força e dos movimentos) através de repouso, medicamentos, orientações e prescrição de exercícios físicos.

O IAMSPE (Instituto de Assistência Médica ao Servidor Público Estadual) é uma instituição pública pioneira no Estado de São Paulo possuindo Serviço de Medicina do Exercício e do Esporte, cujo modelo de atendimento e cuidado baseia-se na prescrição de exercícios físicos personalizados e adequados para a promoção e prevenção primária à saúde e também para tratamento de doenças agudas ou crônicas, buscando melhora na qualidade de vida, redução do número de consultas, dos exames de maior complexidade, das visitas ao pronto socorro, das internações e das cirurgias.

O presente estudo realizado nesta instituição busca caracterizar-se apenas a orientação da atividade física pode trazer benefícios aos pacientes com OA avançada de joelho, reduzindo limitações e manifestações clínicas desta patologia e contribuindo para a melhora da qualidade de vida.

\section{MATERIAIS E MÉTODOS}

\section{Amostra}

Pacientes com diagnóstico de $\mathrm{OA}$ avançada e indicação formal de artroplastia do joelho acometido, que procuraram o Ambulatório de Medicina Esportiva e Exercício do HSPE-IAMPSE, localizado na cidade de São Paulo, Brasil.

\section{Desenho do estudo e definições}

Estudo prospectivo de intervenção.

\section{Critérios de inclusão}

Pacientes com OA primária avançada do joelho, com indicação cirúrgica de artroplastia total do joelho, apresentando grau III, IV ou V da Classificação de Ahlbäck ${ }^{5}$ modificada por Keyes et al. ${ }^{6}$. Foi definido o grau III de Ahlback como estágio não avançado (moderado) e os graus IV e V como estágios avançados;

- Pacientes com idade superior a 50 anos de idade;

- Pacientes que não praticavam atividade física formal;

- Pacientes que concordaram em participar da pesquisa;

- Pacientes sem comorbidades que impossibilitassem a realização de atividade física;

- Pacientes que não faziam uso de analgésicos.

\section{Critérios de exclusão}

Pacientes que praticavam atividade física;

Pacientes que tinham alguma patologia pré-existente no joelho, exceto $\mathrm{OA}$

\section{Avaliação dos pacientes}

O estudo foi dividido nas seguintes etapas: na primeira fase, o paciente foi avaliado pelo médico, sendo aplicados a ficha de avaliação e os questionários. Nessa mesma ocasião, além da realização do exame físico foi solicitado o exame radiográfico nas incidências anteroposterior e perfil com carga monopodálica do joelho acometido para a classificação do grau da OA pela Classificação de Ahlback modificada por Keyes et al..$^{5-7}$. Após esses procedimentos, o paciente retornou com o resultado do exame radiográfico ao médico, que o informou sobre como seria realizado o estudo, esclareceu suas dúvidas e obteve a assinatura do Termo de Consentimento Livre e Esclarecido. O presente trabalho também foi submetido e aprovado pelo Comitê de Ética em Pesquisa do IAMSPE, sob o número 420679 em 02/08/2013.

Os pacientes foram orientados sobre os exercícios e receberam uma cartilha para realização dos mesmos no domicílio. Tais exercícios visavam promover alongamento e reforço muscular dos membros inferiores, bem como propriocepção e flexibilidade. Retornaram para reavaliação e preenchimento dos questionários com três e seis meses. A qualquer momento do trabalho, os pacientes poderiam procurar o ambulatório, caso tivessem alguma dúvida a respeito dos exercícios ou caso ocorresse alguma intercorrência. Os exercícios foram apenas orientados, mas não supervisionados.

Os questionários utilizados para avaliar os pacientes foram: WOMAC, validado para a língua portuguesa ${ }^{8}$ e o EVA.

O Western Ontario and McMaster Universities Osteoarthritis Index (WOMAC) avalia as variáveis dor, rigidez articular e funcionalidade em 24 itens, sendo um instrumento válido e confiável, específico para OA. O Womac é um questionário composto por três domínios: Seção A - Dor (cinco questões); Seção B - Rigidez (duas questões); e Seção C - Atividade Física (17 questões). As questões devem ser respondidas de acordo com a percepção de dor, rigidez articular e nível de função física (funcionalidade) percebidas pelo voluntário nas últimas 72 horas. Os escores do WOMAC variam de 0 a 4, sendo distribuído da seguinte forma: nenhuma $=0$; leve $=1$; moderada $=2$; severa $=3$; extrema $=4$. Somam-se os valores de cada seção ou domínio, sendo na seção $A$ - Dor, a pontuação mínima esperada $=0$ e a máxima $=20$; na seção B - Rigidez, a pontuação mínima esperada $=0$ e a máxima = 8; e na seção $C$ - Função física, a pontuação mínima esperada $=0$ e a máxima $=68$. Assim é obtida uma pontuação geral mínima de 0 e máxima de 96 pontos $^{8-11}$.

A Escala Analógica Visual (EVA) consiste em uma avaliação subjetiva na qual existe uma linha horizontal com 10 centímetros de comprimento, assinalada em uma de suas extremidades a classificação "SEM DOR" e na outra a classificação "DOR MÁXIMA". O voluntário realiza a marcação com um traço no ponto que representa a intensidade de sua dor ${ }^{12-14}$. O seu uso em pacientes com OA de joelho tem se constituído em um importante e prático instrumento para avaliação da intensidade da sintomatologia álgica ${ }^{15-17}$.

\section{Análise dos dados e tratamento estatístico}

Os dados obtidos com os questionários aplicados aos pacientes foram tabulados e apresentados em forma descritiva bem como foi realizada a análise das respostas obtidas com condições de serem agrupadas quantitativamente. Foi utilizado o modelo linear generalizado (MLG) com medidas repetidas ${ }^{18}$. Nas situações em que houve diferença significante entre os tempos, para a identificação de quais momentos se diferenciaram entre si foram feitas comparações múltiplas, utilizando o teste de Bonferroni ${ }^{19}$. Para todas as comparações foi considerado um nível de significância de 5\%. 


\section{RESULTADOS}

Dos 34 pacientes que iniciaram o estudo apenas 23 completaram o seguimento com predominância entre eles da faixa etária de 60-69 anos, do sexo feminino, do acometimento de ambos os joelhos e dos graus IV e V de OA, segundo o estadiamento de Ahlback (Tabela 1).

Foi observada diferença estatisticamente significante entre as pontuações pelo escore EVA, entre o momento pré-observância da orientação do protocolo de atividade física e aos três e seis meses após seu início, com maiores valores obtidos na admissão. Tal resultado também foi observado quando utilizado o sistema WOMAC em seus três domínios (Figura 1). Estas diferenças foram observadas em ambos os joelhos, com ilustrações referentes ao direito.

Houve diferença de pontuação entre os momentos pré e três meses, pré e seis meses e entre os momentos três e seis meses de atividade física para os valores de EVA e dos domínios de WOMAC, independente do grau de OA. Entretanto, só houve diferença significativa estatisticamente

Tabela 1. Dados gerais relativos aos pacientes portadores de OA avançada do joelho que completaram o protocolo de atividade física.

\begin{tabular}{c|c|c}
\hline \multicolumn{2}{c|}{ Dados } & $\mathbf{n}(\%)$ \\
\hline \multirow{3}{*}{ Idade } & $50-59$ anos & $5(21,7)$ \\
\cline { 2 - 3 } & $60-69$ anos & $11(47,8)$ \\
\cline { 2 - 3 } & $70-79$ anos & $7(30,4)$ \\
\hline \multirow{2}{*}{ Sexo } & Masculino & $6(26)$ \\
\cline { 2 - 3 } & Feminino & $17(73,9)$ \\
\hline \multirow{2}{*}{ Acometimento } & Unilateral & $7(30,4)$ \\
\cline { 2 - 3 } & Bilateral & $16(69,5)$ \\
\hline \multirow{3}{*}{ Grau de OA } & Grau III & $7(17,9)$ \\
\cline { 2 - 3 } & Grau IV & $24(61,5)$ \\
\cline { 2 - 3 } & Grau V & $8(20,5)$ \\
\hline
\end{tabular}

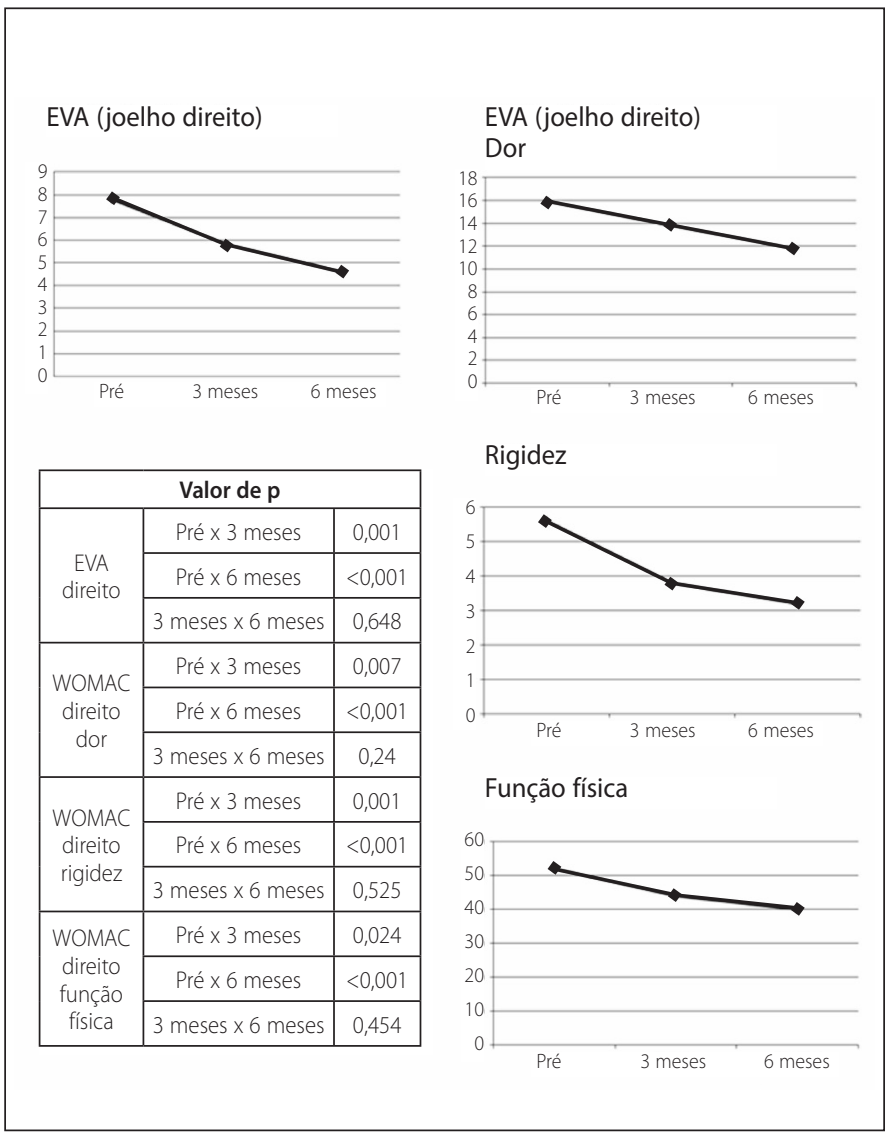

Figura 1. Comparação entre a pontuação EVA e WOMAC para o joelho direito (domínios) nos momentos pré e pós intervenção, no grupo que completou o tratamento. entre os momentos pré e três meses e pré e seis meses ( $p<0,05)$. Maiores pontuações sempre foram apresentadas no momento pré (Figura 2).

As diferenças observadas em relação as pontuações EVA e WOMAC nos momentos pré e aos três e seis meses não sofreram interferência do sexo do paciente ( $P>0,05$ pelo método das comparações múltiplas).

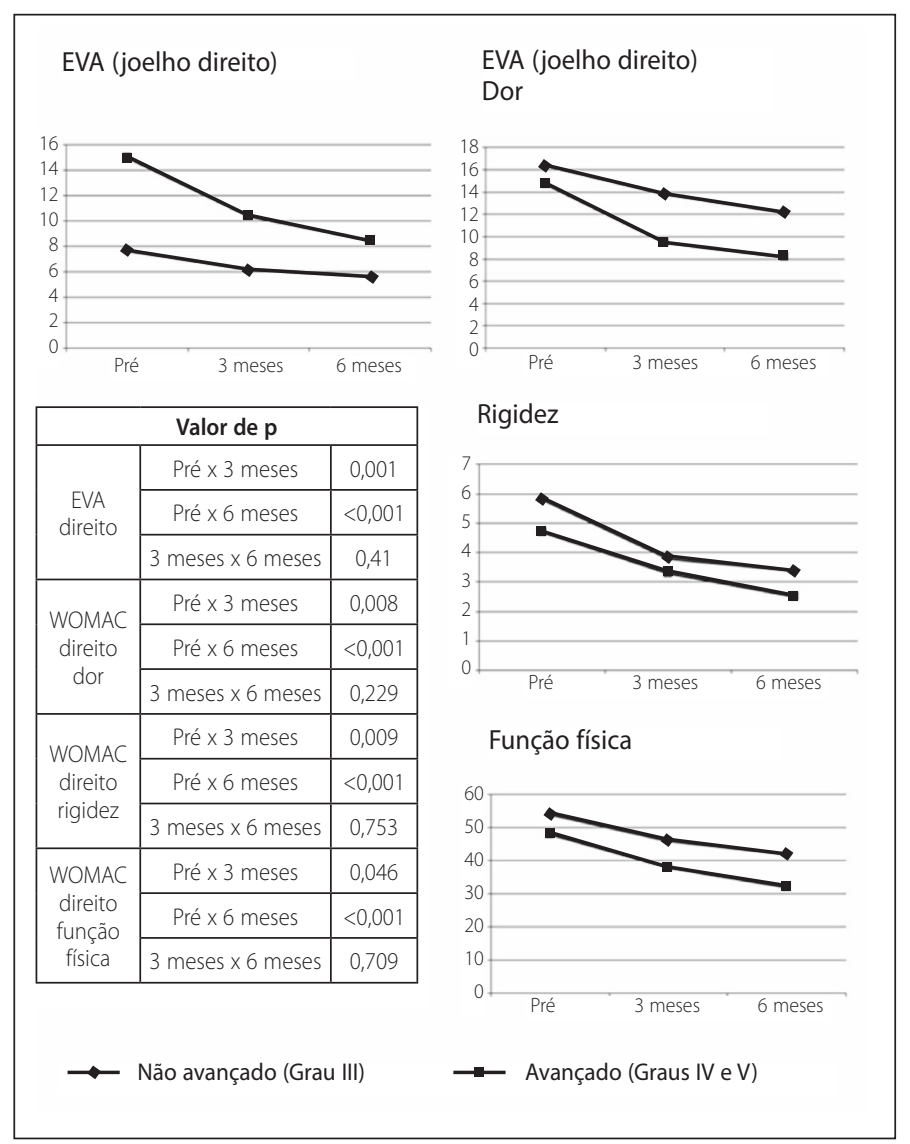

Figura 2. Comparação entre a pontuação EVA eWOMAC (domínios) nos momentos pré e pós intervenção em pacientes com diferentes estágios da doença (Ahlback avançado e não avançado).

\section{DISCUSSÃO}

No presente estudo, um protocolo de atividade física domiciliar foi proposto para pacientes com OA graus III, IV e V, segundo a classificação de Ahlback. Tratou-se de uma amostra de pacientes que se encontravam em uma lista para serem submetidos à artroplastia total do joelho. Foram feitas avaliações aos três e seis meses, sendo franqueado o acesso ao profissional médico em caso de dúvida ou intercorrências. Foi muito elevada a perda de seguimento dos pacientes, apesar de sempre serem contatados por telefone. Também não foi constatada correlação entre o estágio de OA e a conclusão ou não de prática de atividade física orientada. Possíveis explicações para tal fato incluem: a idade elevada dos pacientes; a necessidade de grandes deslocamentos para comparecer à consulta no serviço de referência; e o fato da atividade ser feita no domicílio e não em grupo, o que suprime o benefício do compartilhamento da sua problemática com outros pacientes contribuindo para maior adesão ao tratamento ${ }^{8}$.

Também deve ser ressaltada a importância da monitorização e supervisão rigorosa da atividade física para maximizar seus benefícios clínicos a curto e a longo prazo. No presente estudo a supervisão não foi efetuada, o que pode ter influenciado na adesão dos pacientes ao protocolo proposto, além disso, não se pode comprovar que o paciente realmente realizou os exercícios, sendo a intervenção deste trabalho apenas a orientação de como realizá-los. 
Os 23 pacientes que foram reavaliados após três e seis meses de orientação da atividade física apresentaram diminuição da dor e melhora da função física, confirmada por diferenças estatisticamente significantes entre as pontuações de EVA e WOMAC nos momentos zero e três meses e zero e seis meses. Entre os três e seis meses após a orientação da atividade física também houve melhora das pontuações EVA e WOMAC, porém, sem diferença estatisticamente significativa. A explicação para melhora clínica confirmada pelos sistemas de pontuação utilizados provavelmente deve incluir: ações benéficas do reforço muscular e bem estar mental (melhor motivação). A limitação dos ganhos entre os três e seis meses, ratificada pela ausência de significância estatística, pode dever-se à doença articular definitiva e avançada.

Os ganhos com atividade física no tratamento da dor do joelho são comparáveis aos obtidos com o uso de analgésicos e anti-inflamatórios não hormonais ${ }^{8}$. Deve também ser ressaltada a relação inversa entre a massa muscular, a força muscular e outras medidas de função física com a inflamação em idosos. Portanto o benefício da atividade física pode ser atribuído também à redução da inflamação crônica em idosos ${ }^{19}$. A atividade física também propicia melhor controle das consequências psicossociais da OA. Os participantes aprendem sobre a sua condição, aprendem a aceitá-la, aderem à estratégia permitindo uma melhora de sua condição clínica ${ }^{14}$, o que em nosso estudo foi demonstrado pelo alívio da dor, ganho na função física e diminuição da rigidez.

Finalmente o efeito da atividade física sobre a saúde geral do paciente pode também justificar a melhora da dor e da função física ${ }^{8}$.

As pontuações EVA e WOMAC pré e pós atividade física também diminuíram significativamente e sem relação com o estágio da doença. Este achado pode ser atribuído ao pequeno número de casos representados pelo estágio III, 17,9\% (7/39) dos joelhos acometidos.

Duas exceções foram documentadas. A primeira diz respeito à variação WOMAC dor em joelho esquerdo, quando analisado o estágio da doença (não avançado = grau III e avançado = graus IV e V da Classificação de Ahlback), onde foi verificada diferença significativa entre o momento pré e aos seis meses, independente do estágio de doença.
Uma possível explicação seria tratar-se do membro não dominante. A segunda diz respeito à variação WOMAC função física em joelho esquerdo, onde apesar da queda dos valores não houve diferença significativa entre o momento pré e os três e seis meses e que poderia ter a mesma tentativa de explicação.

A presença de doença uni ou bilateral não interferiu na resposta ao protocolo de atividade física avaliada pelos sistemas de pontuação EVA e WOMAC. Aqui novamente a avaliação WOMAC dor em joelho esquerdo só mostrou diferença estatística entre o momento pré e aos seis meses, podendo ser explicada pela não dominância do membro esquerdo todos os pacientes eram destros. Já o WOMAC função física em joelho direito e esquerdo só mostrou diferença significante entre os momentos pré e aos seis meses, provavelmente refletindo a necessidade de maior tempo de atividade física para que se tenha repercussão consistente na função das referidas articulações.

O presente estudo ilustrou que a orientação da atividade física tem um efeito na melhora da qualidade de vida e funcional dos pacientes com OA de joelho. Possivelmente as condições psicossociais do acolhimento e da orientação em si podem ser consideradas as responsáveis por essa melhora. Apesar do pequeno tamanho da amostra e dela ter incluído pacientes com estágio mais avançado de OA ainda assim se conseguiu confirmar esta associação, persistindo o desafio de ampliar o número de pacientes a serem acompanhados e por um período de tempo mais longo.

\section{CONCLUSÃO}

Conclui-se que a orientação da atividade física no tratamento de pacientes com OA avançada de joelho, com base na avaliação aos três e seis meses dos valores de EVA e WOMAC pré e pós-intervenção, melhora a condição desses pacientes e deve ser considerada, inclusive nos casos de OA com indicação de artroplastia.

Todos os autores declararam não haver qualquer potencial conflito de interesses referente a este artigo.

CONTRIBUIÇÕES DOS AUTORES: Cada autor contribuiu individual e significativamente para o desenvolvimento do manuscrito. RSCF (0000-0002-8195-2227)* foi responsável pelo acompanhamento dos pacientes, orientação da atividade física e aplicação dos questionários. MPN (0000-0001-5892-2532)* avaliou os dados obtidos, incluindo a análise estatística e revisão do manuscrito. Os dois autores contribuíram com o conceito intelectual do estudo. *Número ORCID (Open Researcher and Contributor ID).

\section{REFERÊNCIAS}

1. King LK, Birmingham TB, Kean CO, Jones IC, Bryant DM, Giffin JR. Resistance training for medial compartment knee osteoarthritis and malalignment. Med Sci Sports Exerc. 2008;40(8):1376-84.

2. Sevick MA, Miller GD, Loeser RF, Williamson JD, Messier SP. Cost-effectiveness of exercise and diet in overweight and obese adults with knee osteoarthritis. Med Sci Sports Exerc. 2009;41(6):1167-74

3. Sevick MA, Bradham DD, Muender M, Chen GJ, Enarson C, Dailey M, Ettinger WH Jr. Cost-effectiveness of aerobic and resistance exercise in seniors with knee osteoarthritis. Med Sci Sports Exerc. 2000;32(9):1534-40.

4. Griffin TM, Guilak F. The role of mechanical loading in the onset and progression of osteoarthritis. Exerc Sport Sci Rev. 2005;33(4):195-200

5. Ahlbäck S. Osteoarthrosis of the knee. A radiographic investigation. Acta Radiol Diagn (Stockh). 1968:(Suppl 277):7-72

6. Keyes GW, Carr AJ, Miller RK, Goodfellow JW. The radiographic classification of medial gonarthrosis. Correlation with operation methods in 200 knees. Acta Orthop Scand. 1992;63(5):497-501.

7. Hochberg MC, Chang RW, Dwosh I, Lindsey S, Pincus T, Wolfe F. The American College of Rheumatology 1991 revised criteria for the classification of global functional status in rheumatoid arthritis. Arthritis Rheum. 1992;35(5):498-502

8. Fernandes MI. Tradução e validação do questionário de qualidade de vida específico para osteoartrose WOMAC (Western Ontario McMasterUniversities) para a língua portuguesa [dissertação]. São Paulo: Universidade Federal de São Paulo; 2003.

9. Maly MR. Linking biomechanics to mobility and disability in people with knee osteoarthritis. Exerc Sport Sci Rev. 2009;37(1):36-42.

10. Bellamy N, Buchanan WW, Goldsmith CH, Campbell J, Stitt LW. Validation study of WOMAC: a health status instrument for measuring clinically important patient relevant outcomes to antirheumatic drug therapy in patients with osteoarthritis of the hip or knee. J Rheumatol. 1988;15(12):1833-40.

11. Tanner SM, Dainty KN, Marx RG, Kirkley A. Knee-specific quality-of-life instruments: which ones measure symptoms and disabilities most important to patients? Am J Sports Med. 2007;35(9):1450-8.

12. Huskisson EC, Melzack R. Visual analogue scales. In: Melzack R, editor. Pain measurement and assessment. New York: Raven Press; 1983. p. 33-7.

13. Coleman S, Briffa NK, Carroll G, Inderjeeth C, Cook N, McQuade J. A randomised controlled trial of a self-management education program for osteoarthritis of the knee delivered by health care professionals. Arthritis Res Ther. 2012;14(1):R21.

14. Jansen MJ, Hendriks EJ, Oostendorp RA, Dekker J, De Bie RA. Quality indicators indicate good adherence to the clinical practice guideline on "Osteoarthritis of the hip and knee" and few prognostic factors influence outcome indicators: a prospective cohort study. Eur J Phys Rehabil Med. 2010;46(3):337-45.

15. Pelletier D, Gingras-Hill C, Boissy P. Power training in patients with knee osteoarthritis: a pilot study on feasibility and efficacy. Physiother Can. 2013;65(2):176-82.

16. Salacinski AJ, Krohn K, Lewis SF, Holland ML, Ireland K, Marchetti G. The effects of group cycling on gait and pain-related disability in individuals with mild-to-moderate knee osteoarthritis: a randomized controlled trial. J Orthop Sports Phys Ther. 2012;42(12):985-95

17. Hafez AR, Al-Johani AH, Zakaria AR, Al-Ahaideb A, Buragadda S, Melam GR, et al. Treatment of knee osteoarthritis in relation to hamstring and quadriceps strength. J Phys Ther Sci. 2013;25(11):1401-5.

18. Nelder J. Generalized Linear Models. J R Stat Soc. 1972;135:370-84.

19. Neter J. Applied linear statistical models. 4th ed. New York: McGraw-Hill; 1996. 